\title{
Modelling and control of chaotic processes through their bifurcation diagrams generated with the help of recurrent neural network models. Part 2: An industrial study
}

\author{
J. Krishnaiah *, C.S. Kumar, M.A. Faruqi \\ Robotics and Intelligent Systems Laboratory, Mechanical Engineering Department, Indian Institute of Technology, Kharagpur 721302, India
}

Received 1 September 2003; received in revised form 9 January 2004; accepted 14 April 2005

\begin{abstract}
Many real-world processes tend to be chaotic and are not amenable to satisfactory analytical models. It has been shown here that for such chaotic processes represented through short chaotic noisy observed data, a multi-input and multi-output recurrent neural network can be built which is capable of capturing the process trends and predicting the behaviour for any given starting condition. It is further shown that this capability can be achieved by the recurrent neural network model when it is trained to very low value of mean squared error. Such a model can then be used for constructing the bifurcation diagram of the process leading to determination of desirable operating conditions. Further, this multi-input and multi-output model makes the process accessible for control using open-loop/closed-loop approaches or bifurcation control, etc.
\end{abstract}

(c) 2005 Elsevier Ltd. All rights reserved.

Keywords: Bifurcation diagram; Recurrent neural networks; Multi-variate chaotic time-series; Chaotic process

\section{Introduction}

Many real-world process plants at times behave in an odd way, that is with slight or no practical change in input conditions, large variations in outputs are produced [1-3] or the outputs oscillate between large values not directly related to changes in inputs of the previous time epochs. These plants often have memories of past (due to physical retention of the parts of the outputs, etc.) and have non-linear relationships between input and outputs variables, which have broadly been classified as non-linear or chaotic systems. Control methods for these systems are usually devised differently using nonlinear dynamics or chaos based approaches as compared to conventional approaches.

\footnotetext{
DOI of original article: 10.1016/j.jprocont.2005.04.002

* Corresponding author.
}

Modelling of chaotic systems may be attempted as continuous or discrete dynamical system depending on the application. While complex non-linear multi-input and multi-output non-linear system can be modelled and controlled through new approaches in soft computing and computational intelligence (CI), such techniques have not evolved for chaotic phases of non-linear systems. A chaotic system when modelled as a linear system leads to high predictability errors as has been shown in [4]. At the same time there are important reasons to develop modelling techniques for chaotic systems [5] as these systems have ability to amplify small perturbations, which improves their utility in searching specific desired states with high flexibility and low energy cost. In fact large alterations of desired behaviour are impractical in linear systems, which in contrast in chaotic systems can be achieved by making small time-dependent perturbations, in an accessible parameter of the process. 
Many examples of successful chaotic control of systems do exist in literature e.g., [6-8] where mathematical models can be built for analysis, but for many realworld systems models are difficult to build and thus they remain unapproachable through this route. These systems however remain open for modelling and control through approaches [9] relying directly on the observed data.

However, it has been shown in [10] that it is possible to build a multi-input and multi-output recurrent neural networks model of the system by observing the inputs and outputs of a given system or process and training the network to low levels of mean squared error (MSE) (typically of the order of $10^{-2}$ or better). This multi-input and multi-output (MIMO) model can be used either for bifurcation control or parameter based control of chaos or for implementing other strategies like stabilisation, etc. It has also been demonstrated that these MIMO models are robust against Gaussian noise and thus suggesting that these could be used in noisy process plant environments. The feasibility of the propositions have been demonstrated in [10] using a well known two dimensional discrete chaotic system of Hénon map. It is proposed here to examine the applicability of this technique, through suitable adaptation, for modelling real-world process plants that can operate in stable and chaotic operating conditions.

The process plant selected for evolving and validating the proposition is a submerged arc furnace (SAF) which is used for producing ferroalloys. Its behaviour has been observed to be complex as typically large variations in outputs (which are undesirable) are observed with very small or no changes in the input parameters as shown in Fig. 1. Further, the process is found to be sensitive to process control parameters like power input, etc. as well, giving rise to possibilities that the process can operate in chaotic and non-chaotic modes, etc. Control and charging procedures followed are based on the plant practice and have been kept unchanged during observations. A brief description of the furnace has been provided in Appendix A.

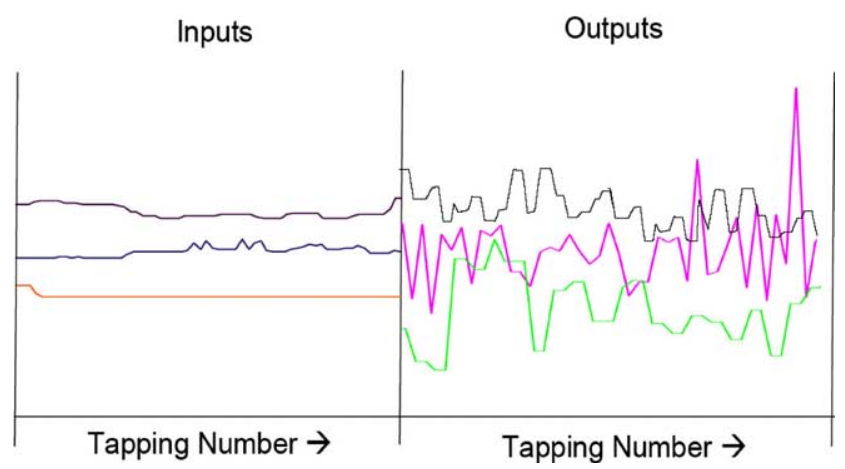

Fig. 1. Typical variations observed in inputs and outputs of the submerged arc furnace.
In a SAF, electrodes are submerged inside the raw materials (ores + fluxes + cokes) and arcs are struck between electrodes and the raw materials to generate large quantities of heat to carry out various reactions. The presence of high temperatures, huge electrical arcs with varying currents, multiple chemical reactions and the fact that a large mass always remains inside the furnace, compared to one which is periodically tapped, makes it a complex and difficult to model process. In [11] it has been shown that the working of SAF can be chaotic in nature, based on the calculated value of the one of the Lyapunov exponents turning out to be positive for one of the output variables evolving in time.

Literature does not provide many instances of controlling chaotic process plants using models built on observed data. The prevailing approach is to resort to delay coordinates embedding [12] of time-series data to determine the attractor and other properties of the system, but this approach does not link all the input variables to output variables and thus is not useful directly for control. Recently De Feo [13] has used a system identification technique based on Genetic Evolution of two part (linear + non-linear) ARMA model trained on chaotic data. While this approach leads to successful classification of slightly differing periodic data, its use in developing any controller has not been demonstrated. In [14] it has been shown that using reinforcement learning techniques, control of chaotic systems can be achieved from observed data based methods without formal models, but the approach has been limited to stabilising an unstable fixed orbit. Therefore, the proposition brought out in [10], where modelling the system through multiinput and multi-output recurrent neural networks, training it to very low MSE levels using back-propagation algorithm, to achieve a capability to predict number of subsequent steps, has been adopted here, for the study of real-world systems. It is to be noted that in long terms all chaotic systems remain unpredictable [4]. The capability of these models in being able to predict number of subsequent values from a starting point, and also learn the behaviour of the system to be able to estimate the Lyapunov exponents of the system, leads to the feasibility of these being used to draw the bifurcation diagram (BD) of the system, with respect to an accessible control (bifurcation) parameter. The BDs constructed can be used for achieving parameter based control of the system as it identifies the chaotic, oscillatory and stable regions of operation of the system. For any fixed value of accessible parameter along the $\mathrm{BD}$, an attractor of the system can be constructed and control and optimisation strategies can be developed based on its shape.

Modelling of real-world chaotic systems raises some additional issues due to the strong dependency of the system evolution on the initial conditions (besides noise), making the availability of long series of observational data difficult as any plant may have to be reset for various 
reasons. However, by adapting a RNN based approach that requires a multi-input and multi-output RNN to be trained first from the data, these difficulties can be avoided as data for RNN training can be in any sequence, thus completely eliminating the need for the having the observations in a predetermined series form. This allows observations to be taken from any running plant without any constraints on the operation of the plant.

Thus, to study the operation and control of SAF, or any plant which is likely to be chaotic, an approach based on the strategies developed in [10] and the issues discussed above the following sequential procedure is adopted:

(I) Using the raw data, obtained from the plant (in any sequence and without noise filtering) comprising of all the available input and output parameters, a MIMO-RNN model is trained.

(II) For the RNN model, an architecture is selected using rules of thumb and experimentation, which should allow the MSE of the network under back-propagation training to be brought to a level of $10^{-2}$ or better.

(III) The above MIMO-RNN model of the plant is validated on real data for single inputs as well as sequential inputs/outputs. (This model is subsequently used for testing all propositions and also acts as a plant simulator.)

(IV) Now using this MIMO-RNN model for any desirable initial conditions the bifurcation diagram of the system is generated for any accessible parameter showing various regions of stable, oscillatory or chaotic operations.

(V) Now for any chaotic or other regions of the operations of the system, for any particular set of initial conditions the largest Lyapunov exponent is computed based on the model developed, and the degree and the nature of chaos is established.

(VI) Next, the bifurcation control strategy is implemented through the input parameter control using the knowledge from the above mentioned model and the possibility of changing the Bifurcation Diagram or the operating point on it, is examined to operate the process in desirable conditions.

(VII) A procedure for reducing the oscillations during the chaotic operation is attempted by changing the attractor of the system through RNN + GA based searches on the inputs.

(VIII) To cater to the needs of the real-world systems, the procedure for stabilising on an attractor, devised to work in presence of noise and of small changes in initial conditions during the operation, is implemented through RNN + GA combined search procedures.

\section{Operation of a submerged arc furnace producing ferroalloys and its input-output data modelling}

\subsection{Modelling the submerged arc ferroalloy making process}

The submerged arc furnace (SAF) selected for the study is used for producing ferroalloys. These alloys are used as alloying elements in producing steels. This SAF produces variety of alloys. In the present case the $\mathrm{SAF}$ is used for producing silico-manganese ( $\mathrm{SiMn}$ ) alloy.

The submerged arc furnace (Appendix A) considered is charged as per the charge balancing procedure based on the chemical reactions and the plant practices. While the furnace runs continuously, the tapping of the output ferroalloy is carried out periodically and analysed. Data from the SAF output is thus collected on tapping-to-tapping basis. The data collected from the plant has different sampling interval for different parameters and also tapping intervals may differ to some extent. For the purpose of modelling, the values of input variables have been averaged on hourly basis. Time unit for modelling has been taken as mean tapto-tap interval. The furnace has been studied while producing ferroalloy of silico-manganese (SiMn-6065) type with nominal composition using a charge mix of cokes, fluxes, Quartz and manganese ores together as inputs (details in Appendix A). The power input to the furnace has also been taken as an input variable for modelling, as the process is known to be sensitive to this. It also provides an easily accessible parameter for control.

The furnace is charged in a way so as to maintain the height of the charge inside. The output is primarily controlled by the input power. Since the energy efficiency of the process keeps changing the alloy output rate is also monitored. The total input power consumed when divided by this quantity in tons yields to specific power consumption. This has also been used as output variable in addition to composition of the alloy and slag, etc. in developing the models.

\subsection{Identifying the chaotic conditions in the plant}

The SAF or other chaotic systems do not always run in chaotic conditions and it is important to know the state at any time to be able to control it. While the proper identification of issues have been addressed in subsequent sections, using proposition outlined in [10], passive identification of chaos has been done by observing the increase of scatter in the outputs, whenever the operation is in the chaotic region. In addition the presence of large oscillations in the outputs without any visible change in input conditions has also been taken as a sign of chaotic conditions in the plant. 


\section{Evolving a suitable RNN architecture and modelling of the plant}

\subsection{Evolving of a suitable RNN architecture}

Based on the studies reported in [10] a RNN architecture shown in Fig. 2 is selected for modelling the submerged arc furnace producing ferroalloys. As mentioned in Appendix A, the SAF operation is a semi-batch operation as a large fraction of the inputs remains inside even after the tapping is done. Thus, the operation of the furnace depends not only on what is

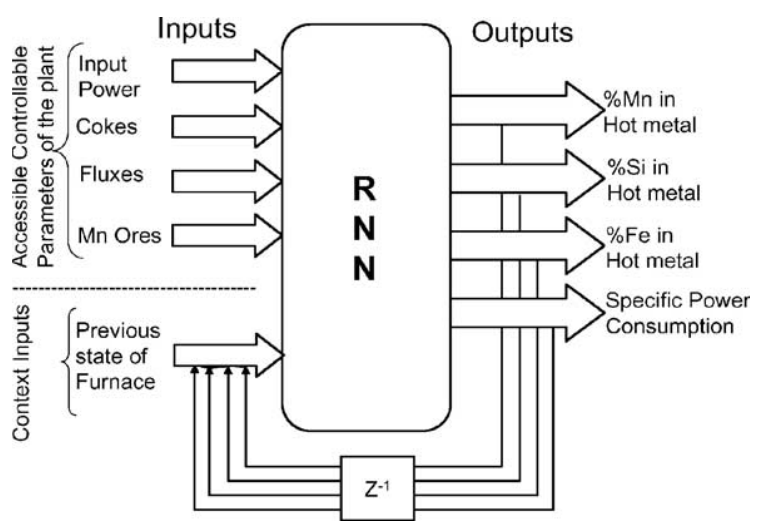

Fig. 2. Block diagram of RNN model for the submerged arc furnace used in the present study. changed now but also on what was charged in the previous time-steps. While developing the model this fact has been accounted for by considering the previous time step values of hot metal composition and the specific power consumption outputs from the plant also as inputs as shown in the block diagram of the model in Fig. 2. This lead to at least one level of recurrence in the model. More levels of recurrences can be provided by using outputs of even earlier time epochs also as inputs.

The RNN architecture and recurrence levels were obtained by trial and error using the broad guide lines suggested in Ref. [15]. Finally, a multi-layer recurrent neural networks architecture has been selected with 18 input and 4 output parameters (relevant in study) of the plant (Appendix A). The level of recurrence is one time step which results into 4 context inputs from the previous time epoch. Thus, the architecture has a total of 22 input (18 actual inputs +4 context inputs) and 4 outputs with one hidden layer consisting of 25 nodes. The training of RNN was carried with 145 data sets, each covering one tapping data during continuous operation of the furnace. A MSE level of 0.009 could be achieved during training with the back-propagation algorithm with 4120 iterations. Thereafter the MSE did not improve.

This RNN model is capable of evolving the system from any desirable initial conditions (charges and power input) provided the previous time epoch outputs of the furnace are also available. Fig. 3 shows actual outputs
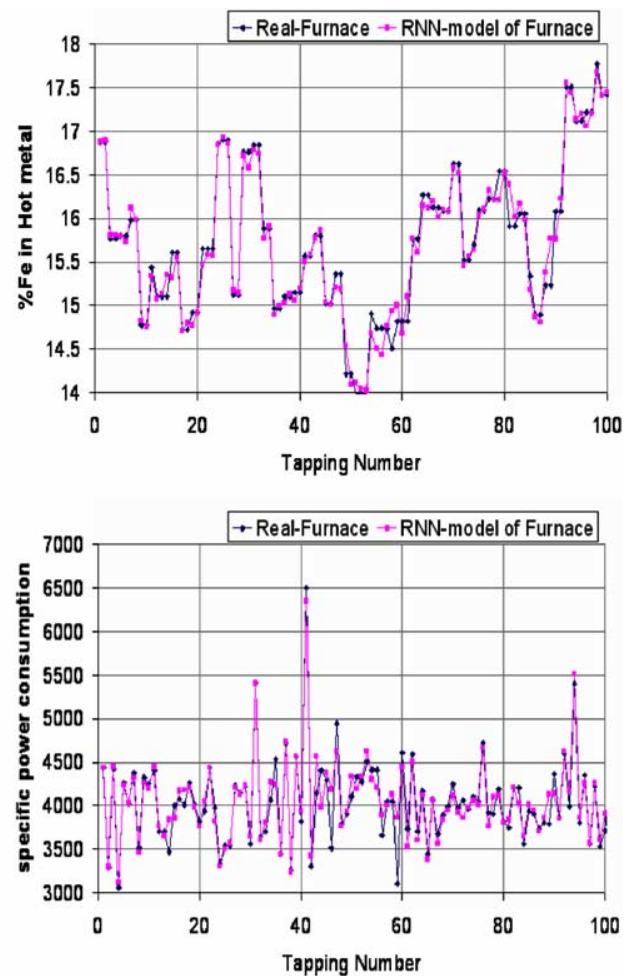

Fig. 3. One step prediction of future outputs of the SAF through RNN models. 
of the furnace compared with one step prediction values of the RNN model. Average prediction error for one step prediction is less than $\pm 1 \%$ and this model can also predict several steps in future. This prediction accuracy is considered to be enough for testing the proposed modelling and control strategy as the real-world plant may have noise and observation errors which may typically be $\pm 1.0 \%$ to $\pm 2.0 \%$.

\subsection{Construction of the bifurcation diagrams}

The BDs constructed from these RNN models using any set of initial conditions showed stability against the number of data sets used and the error level (studied in next section) of training of the RNN model. The BDs have been plotted by generating the data through RNN models, using furnace power (an independent variable) as the control (bifurcation) parameter and keeping all the other inputs constant. A typical BD thus constructed for specific power consumption is shown in Fig. 4. However, the BDs can be constructed for any of the output variables (3 composition variables and specific power consumption) of the RNN model in the present study.

Chaotic systems being dynamical systems the points in the BDs approach the final values through oscillations in the outputs. These oscillations are important from process control point of view. Insets of Fig. 4 show the oscillations as computed from the RNN model for different operating regions. They are further studied in subsequent sections. The oscillations in chaotic zone when plotted in phase-space $\left(X_{t}\right.$ vs. $\left.X_{t+1}\right)$ yield the attractor of the system as the one shown in Fig. 5. The chaotic attractors trajectory patterns define the chaos and those repeat themselves with small variations as the system evolves.

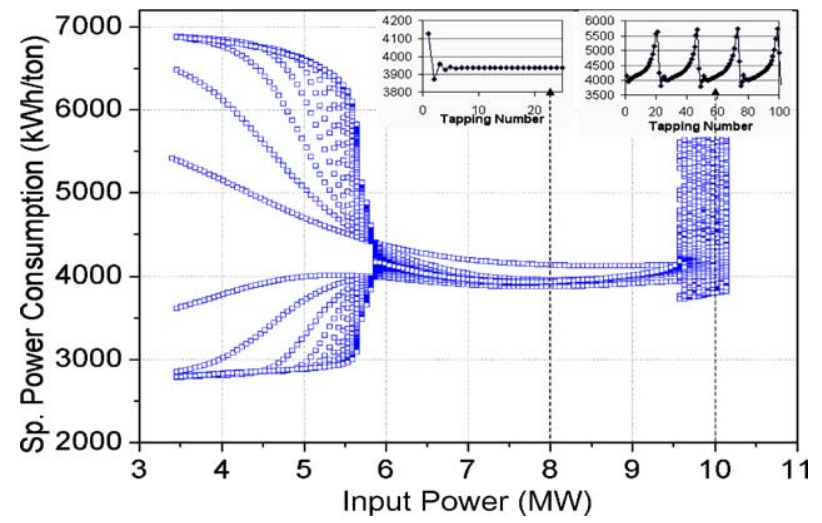

Fig. 4. A typical bifurcation diagram constructed from a RNN model of the SAF. In this diagram the initial points before settling down have also been considered for plotting. For two different cross-sections of the diagram the behaviour of the SAF as predicted by the MINO model in time domain is also shown in inset.

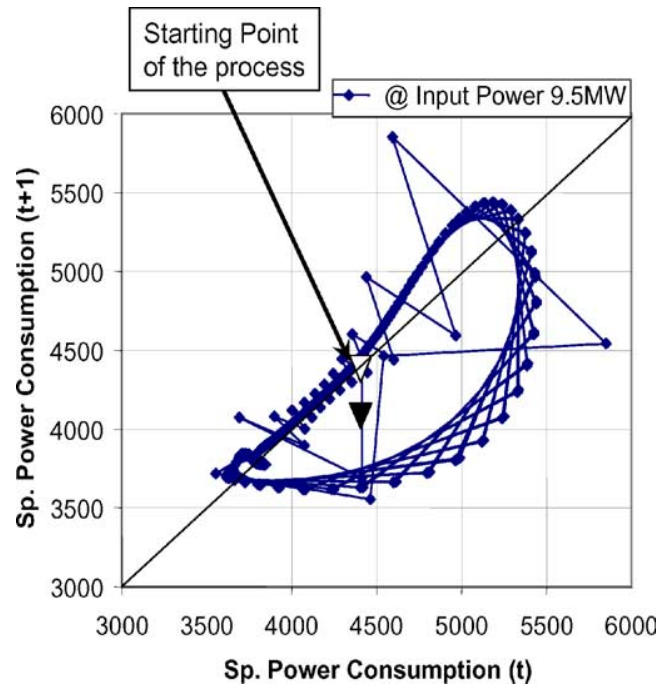

Fig. 5. A phase-space diagram (attractor) for the specific power consumption of SAF at $(t)$ and $(t+1)$ tappings when the power input is $9.5 \mathrm{MW}$ for a typical input composition. Unfilled triangle shows the starting condition of the SAF and the arrow shows the direction in which it moves.

\subsection{Effect of MSE of the network on the bifurcation diagrams}

To examine the stability of the BDs with improving MSE levels of the trained RNN, plots such as shown in Fig. 6 have been developed. It can be seen that the shape of BDs and associated attractors do not change after MSE of 0.01 (for this architecture) but the bifurcation point keeps moving against the control parameter values till MSE values are much lower. It has also been observed that it does not change after the MSE level of nearly 0.009 is achieved. This observation is similar to one observed for Hénon map in [10]. The fact that the attractor becomes visible quite early even when the MSE of RNN is quite coarse.

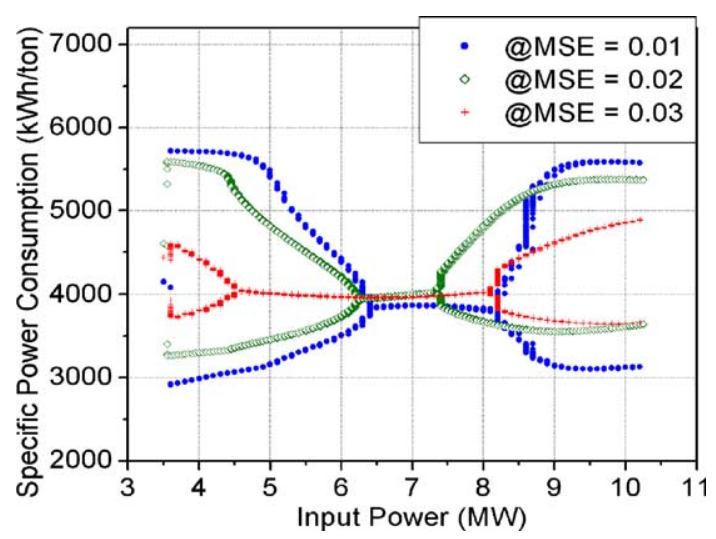

Fig. 6. Evolution of the BDs generated by different MSE level recurrent neural networks models of the SAF. The evolution of bifurcation diagrams is from coarser MSEs to finner MSEs. The evolution stops with reduction in MSE. 


\subsection{Validating the MIMO-RNN model of the process}

The ability of MIMO-recurrent neural networks model developed has been tested for one step prediction and also several steps prediction from the starting point. As shown earlier in Fig. 3, for one step prediction of output variable of the plant and the error of prediction on an average has a value of less than $\pm 1 \%$. Testing the model for predicting several future values from a given initial condition was not possible as the plant input conditions are not maintained at fixed values. Therefore, the prediction of next several steps from a given initial conditions was carried-out, allowing for changes in inputs, but without taking any feedback of real values from the plant. Fig. 7 shows such plots on several output variables. It can be seen that predictions are close to actual values but the results become poor (not shown in the figure) after 20 steps as could be expected in a chaotic system [4].

However, there are regions in plant operation when the inputs are not changed significantly and a plot is shown in Fig. 8 shows the six successive outputs predicted from starting point; which are found to be close to the observed values. The low prediction errors of the MIMO-RNN model on one step prediction and their capability to predict several future steps make the model suitable for studying chaos in the system and

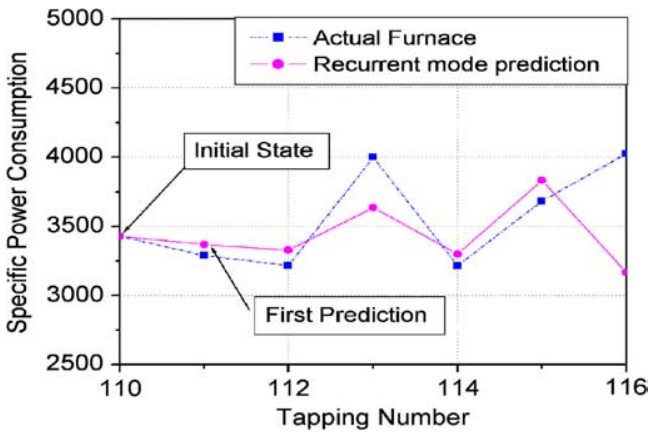

Fig. 8. Predictions of future values of outputs from a starting point computed through RNN model and compared with the actual values. The input condition occurring at tap number 110 remain unchanged in the plant till tap number 116 .

devising control procedures. It should also be noted that the RNN models developed have only one step of recurrence and thus knowing the current output is enough to evaluate the consequences of new inputs.

\section{Controlling a chaotic process by altering its bifurcation diagram through input parameters}

While controlling chaotic systems through stabilisation of an unstable periodic orbit remains important
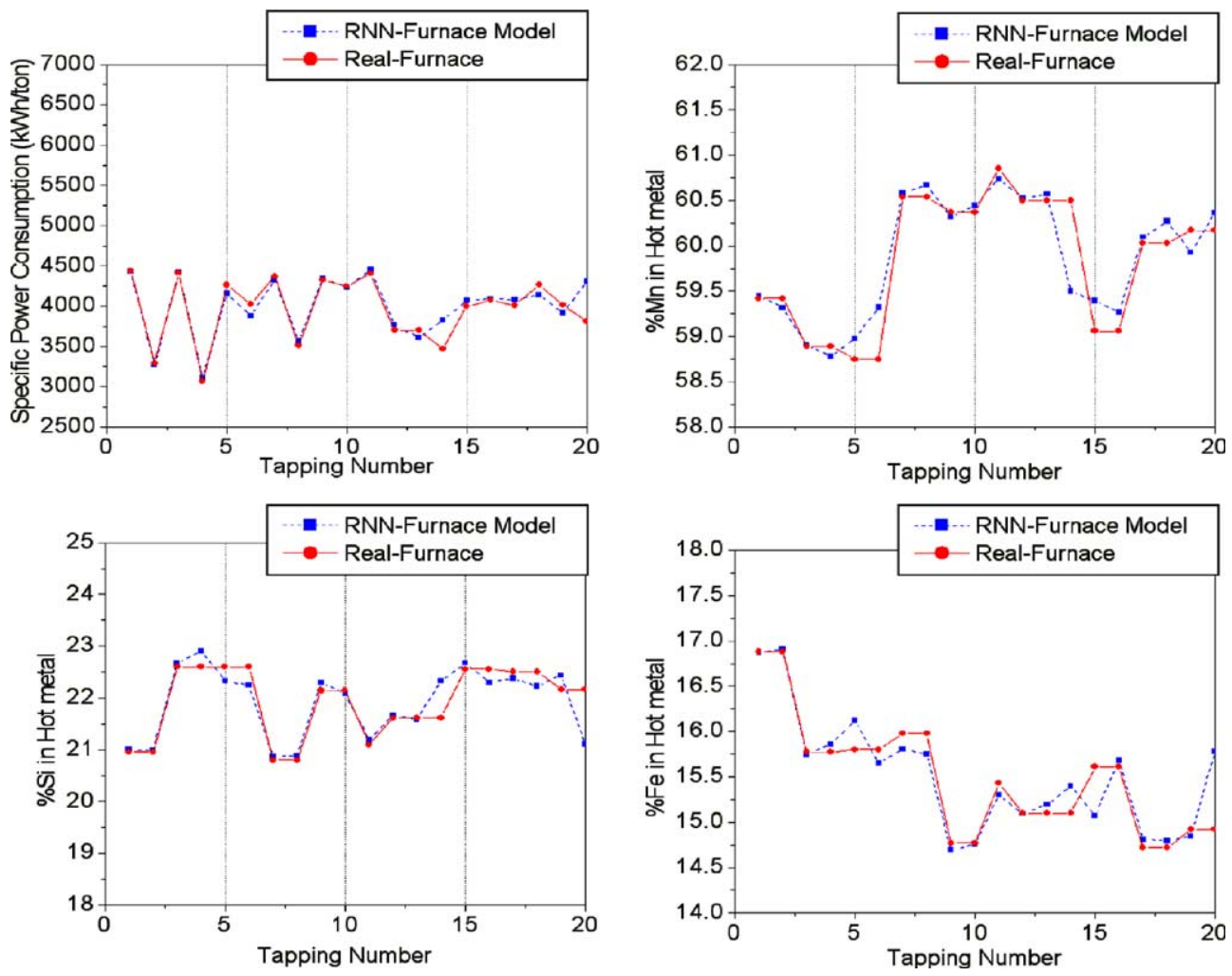

Fig. 7. Prediction of future outputs of the SAF through RNN model from a starting point with inputs modified as per observations without any feedback of outputs from the plant. The predicted values are compared with actual observed values. 
method of chaos control [14], but as studied in [10] the method requires that each orbit be observed while waiting for particular type of orbit to occur. This further requires modification in input parameters through which the operating point on the attractor of the system can be shifted to a stable region to meet the desirable objective. Further, to use it for the real-world process it is required that it should be robust against noise, should be capable of real-time performance, and when the process is being carried-out no undesirable outputs should be produced. These requirements make it difficult to implement it for process control. However, this approach is studied further in Section 5. An approach of using input parameters to change the bifurcation point of the $\mathrm{BD}$ has been studied in this section as it avoids many of the above difficulties.

The multi-input and multi-output chaotic RNN model of the process developed in this study allows the RNNs to be trained for all possible conditions of the operation of the process plant and thus allows the $\mathrm{BD}$ to be constructed for any particular set of starting values. This feature thus helps in finding the desirable operating conditions and in stable or other regions of operation by shifting the bifurcation point through the accessible control parameters. It may also allow searching for optimum operating conditions, etc.

Fig. 9 shows four different randomly generated BDs from a single MIMO trained RNN model, with different (but permissible inputs in terms of charge balance values, etc.) starting inputs (charge compositions and input power). The system may continue to evolve along any of the BDs if the input parameters are kept constant. It can thus be seen that there are very large number of possibilities along which the system can evolve. For example it can be seen that by adopting the composition as charged in Fig. 9(d), there will be chaotic working region at high power input and by adopting composition as charged in Fig. 9(c) more stable and low specific power requirement conditions can be created.

To support the above ideas three different but slightly differing actual operating situations have been selected from the observed data and their BDs have been plotted through the trained RNN as shown in Fig. 10. The BDs

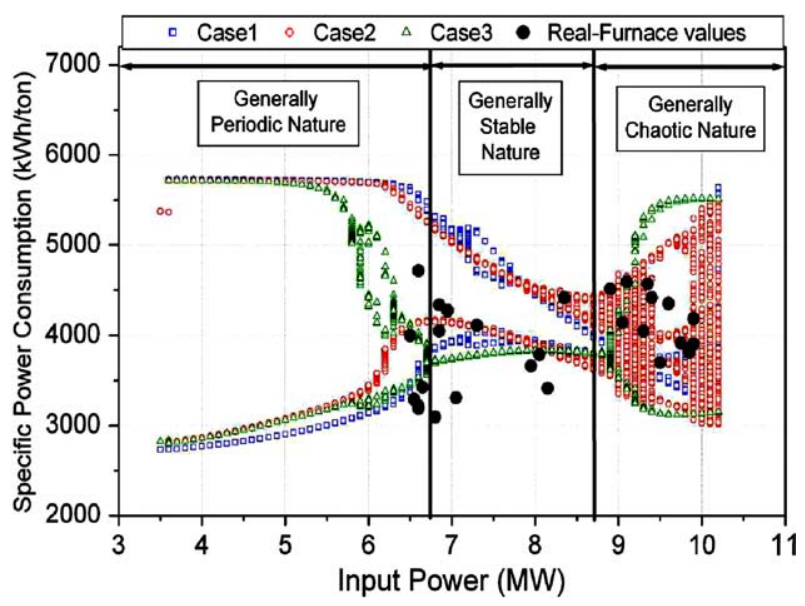

Fig. 10. Points of actually observed values of specific power consumption in SAF (operated at different power levels but with similar composition inputs) plotted on the bifurcation diagrams of the system generated through RNN models. The values are qualitatively different in each region of the operation suggesting their nature of the operation.
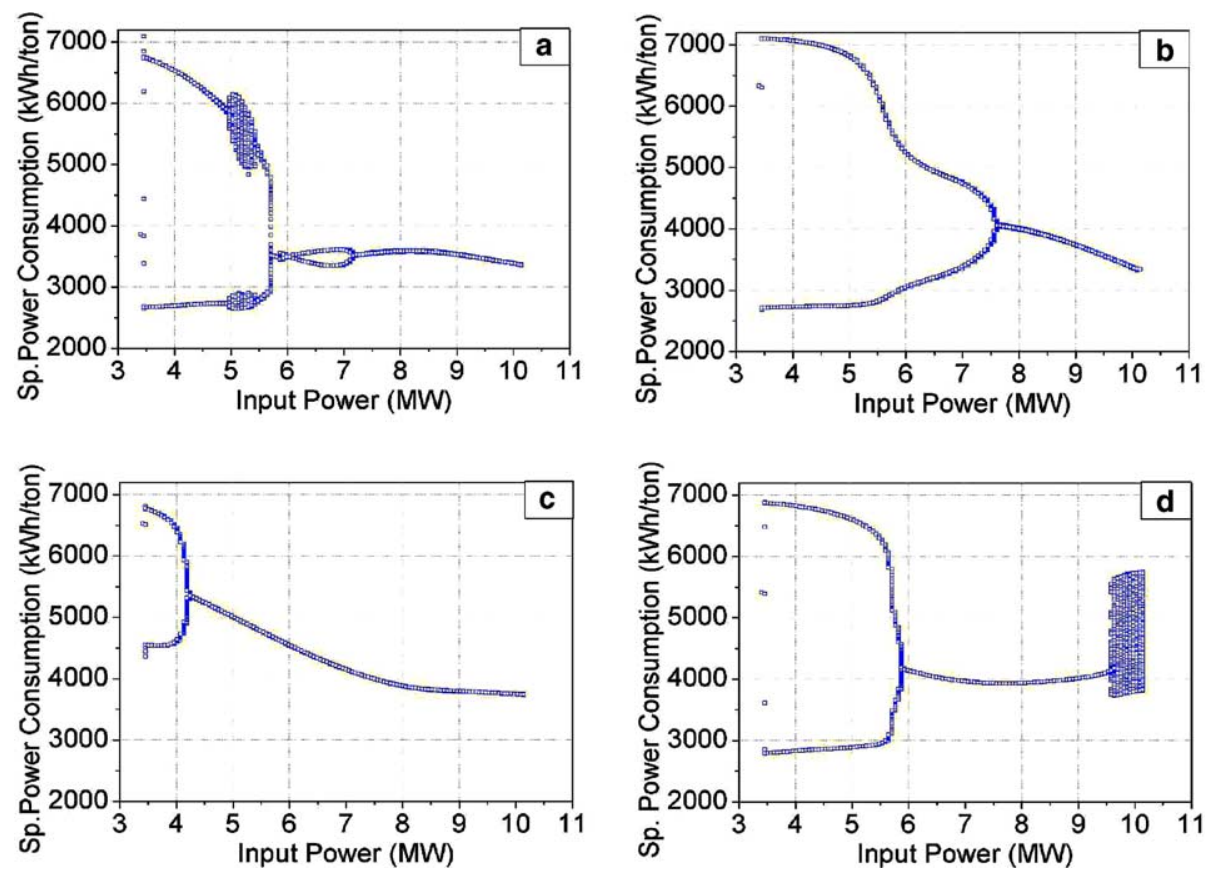

Fig. 9. Typical bifurcation diagrams of specific power consumption in SAF with respect to power input 
are largely similar to each other and the predicted ranges for stable, oscillatory and chaotic regions are also roughly similar. On this diagram the actually observed values of specific power consumption (one of the outputs of the RNN model) have also been plotted when the furnace is charged with similar composition inputs, but the furnace operation is carried out at different input power levels. It can be seen from the diagram that these specific power values fall in different regions of the diagram. For example, when the operation is in chaotic region, variability in values of the specific power consumption is higher as compared to when the operation is in stable region, which can be expected from the nature of the regions.

\subsection{Estimating changes in the output composition variables and the bifurcation point with changes in inputs}

The utility of general purpose RNN model developed can be established by first estimating the variations in the output variables with changes in the inputs when the process runs in either stable or chaotic conditions, and then comparing it with real observed values. An example in Fig. 11 shows the bifurcation diagram for a particular set of initial conditions with respect to input power to the plant. From the diagram it can be seen that under stable conditions of operation at $7 \mathrm{MW}$ the $\% \mathrm{Mn}$ will be close to $60.5 \%$ and under chaotic conditions (around 9.5 MW) it will be in the range of 58.5-62.5\%. This compares well with the observed values of 60 $61 \%$ for stable domain and the range of $59-62 \%$ for the chaotic domain.

Further, the variations predicted from the RNN model (for the same input conditions used as in Fig. 11) for $\% \mathrm{Fe}$ in hot metal and the specific power consumption are shown against the input power in the Figs. 12 and 13 , respectively and are found to be close to the

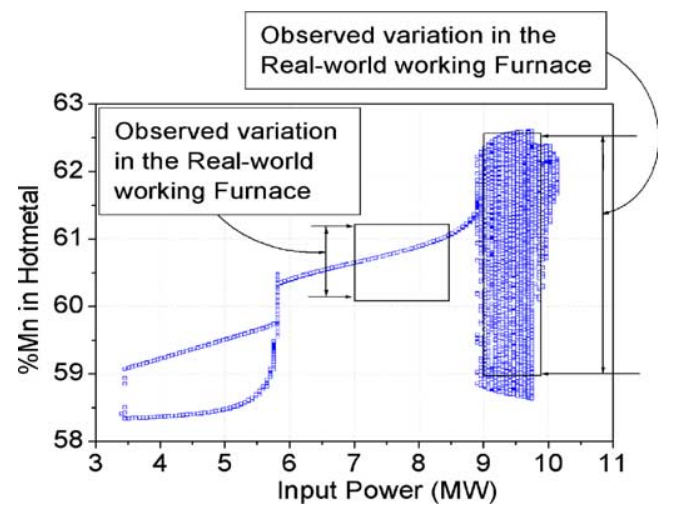

Fig. 11. Variation in $\% \mathrm{Mn}$ in the hot metal as predicted by the $\mathrm{BD}$ of he process for a given set of input conditions, compared with the range of actually observed values in the stable and chaotic regions.

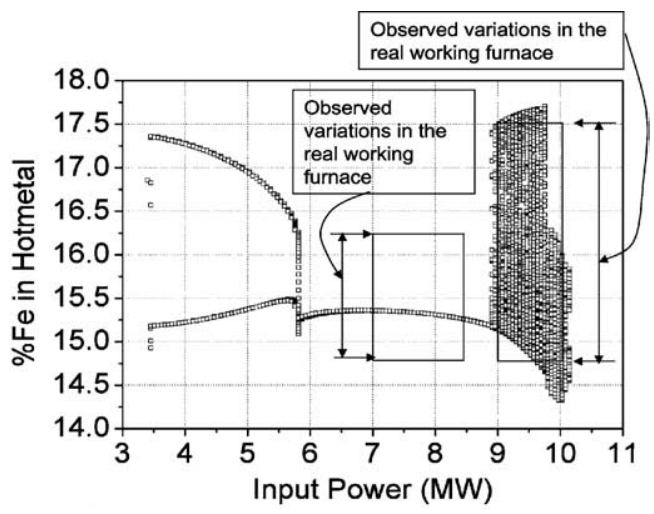

Fig. 12. Variation in $\% \mathrm{Fe}$ in the hot metal as predicted by the $\mathrm{BD}$ of the process for a given set of input conditions, compared with the range of actually observed values in the stable and chaotic regions.

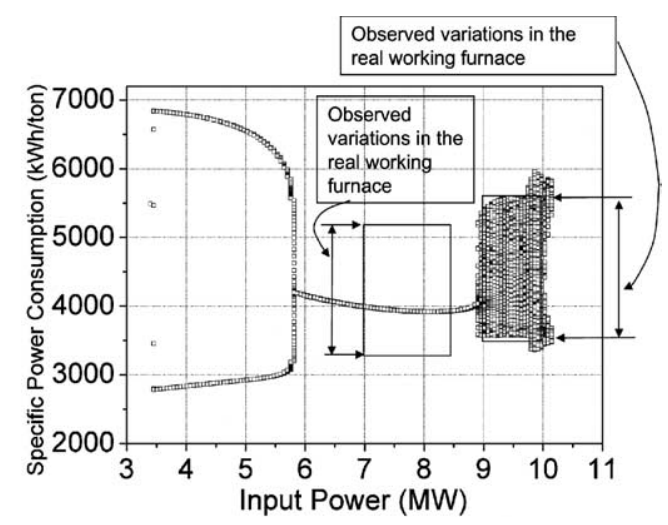

Fig. 13. Variation in specific power consumption as predicted by the $\mathrm{BD}$ of he process for a given set of input conditions, compared with the range of actually observed values in the stable and chaotic regions.

actually observed values, confirms the utility of the model for predictions.

The RNN models developed can as well also be used for studying the effect of composition variables on the BDs. A case for the composition used in Fig. 11 is shown in Fig. 14 where Quartz input has been both increased and also decreased from the starting value. It can be seen that the chaotic region in the BD is shifted and eliminated all together with an increase in Quartz content of the input charge.

The model developed can also be used for drawing the bifurcation diagram of the system using any of the inputs as bifurcation parameter. Fig. 15 shows typical bifurcation diagrams drawn using Quartz in charge as a bifurcation parameter.

This is a useful proposition, since Quartz is a reductant and as well as a fluxing agent in the silico-manganese furnace and considerable variations in Quartz input are permissible. 


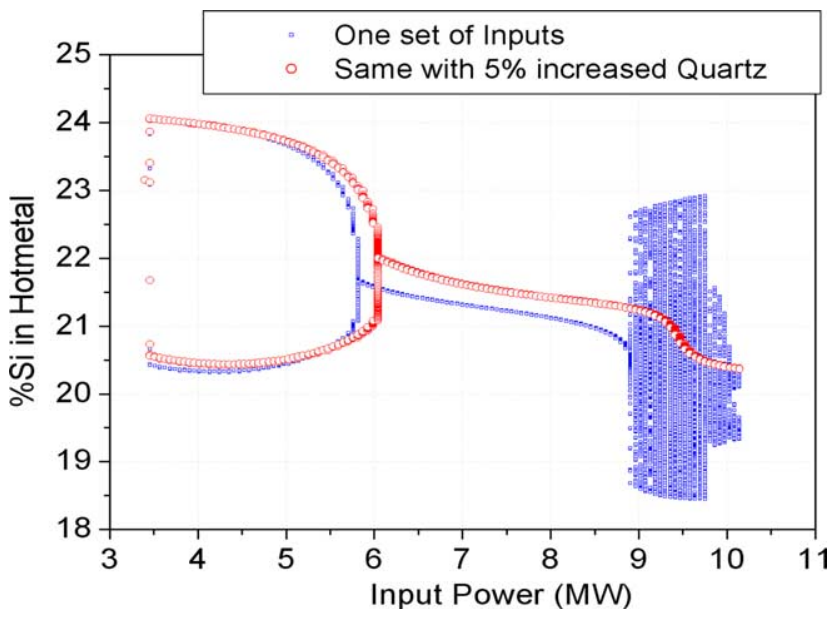

Fig. 14. Bifurcation diagram for $\% \mathrm{Si}$ in hot metal with a given set of inputs, one with normal Quartz content and the other with 5\% increased content. The chaotic zone is eliminated with the increased Quartz content.

\section{Controlling a chaotic process through its attractor}

\subsection{Visualising the attractor}

The attractor(s) of a given chaotic industrial system needs to be visualised before any control strategy may be decided. For any set of initial starting conditions (comprising of current input charges and the previous state of the plant in the case of SAF) the attractor of the process can be constructed in phase-space by observing successive values of an output parameters predicted through the developed MIMO-RNN model. This visualisation may be done just before the particular control strategy is adopted, assuming computation time is available between the observations and implementation on the plant. This is generally the case for slow moving chemical and metallurgical processes, including the one selected here.
A typical attractor for the SAF is plotted in Fig. 5 using the procedure outlined above, for specific power consumption (one of the outputs of the model) during simulated operation in the chaotic zone. The many-toone property displayed by the attractor is a common feature of chaotic systems. It implies that for the same input conditions a varying set of outputs can be produced by the system. The variation occurs in a periodic fashion as shown in Fig. 5. The attractor shape depends primarily on initial conditions.

Further, a $45^{\circ}$ line when drawn on the attractor diagram shows an upper and a lower region of intersections. They act like fixed points as the subsequent outputs will change very little when operating conditions are close to these. From Fig. 5 it follows that the specific power will change very little in subsequent steps in these region and will be lowest when operating in the lower region.

\subsection{Controlling a chaotic process by changing its attractor}

The approach adopted is broadly based in the proposition made in [10], where it has been suggested that by small changes in input parameters often the chaotic systems' attractor can be changed significantly and used for driving the process to more desirable directions. From the studies on the SAF it is seen that it is indeed so and even small changes in input charges and applied power can create a new attractor as shown in Fig. 16. Some of these attractors may be more compact (lesser magnitude of variations) or may take the process to more desirable operating condition like lesser specific power consumption, etc. The alternative attractor shown in the figure has been searched using the furnace simulator (Appendix A). The simulator allows on screen visualisation of bifurcation diagrams of all outputs along with their attractors in real-time as the SAF inputs are altered for searching. A typical search needs to be

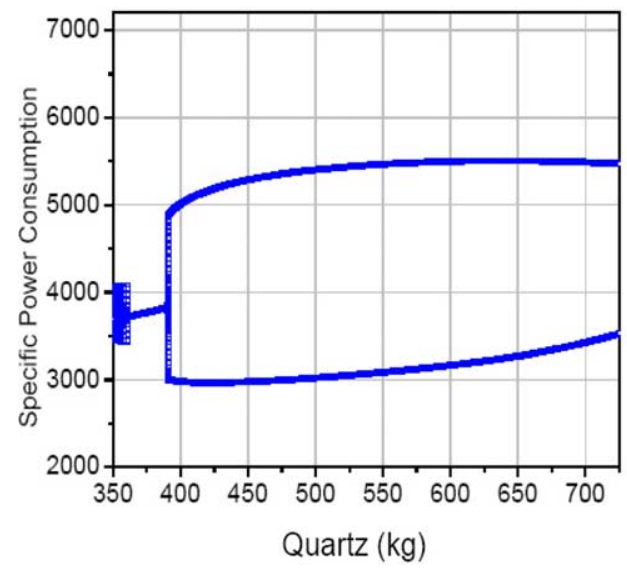

Fig. 15. Typical bifurcation diagrams drawn using Quartz as bifurcation parameter. 


\begin{tabular}{|c|c|c|c|}
\hline & Name & Charge-A & Charge-B \\
\hline 4 & Power & 9 & 9 \\
\hline \multirow{16}{*}{ 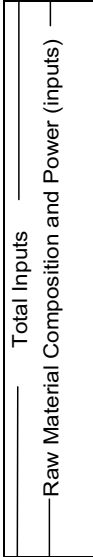 } & Coke1 & 701.5 & 674.1 \\
\hline & Coke2 & 635.42 & 892.72 \\
\hline & Coke3 & 0 & 0 \\
\hline & Coke4 & 0 & 962.48 \\
\hline & Quartz & 355.83 & 607.29 \\
\hline & DBIstGr & 335.5 & 236.85 \\
\hline & Dolamit & 1977.42 & 2629.57 \\
\hline & MnOre1 & 0 & 0 \\
\hline & MnOre2 & 1728.33 & 1821.88 \\
\hline & MnOre3 & 508.33 & 147.92 \\
\hline & MnOre4 & 0 & 0 \\
\hline & MnOre5 & 790.92 & 522.27 \\
\hline & MnOre6 & 388.12 & 0 \\
\hline & MnOre7 & 0 & 0 \\
\hline & MnOre8 & 0 & 0 \\
\hline & Remelt & 0 & 0 \\
\hline$\nabla$ & PF & 0.8 & 0.81 \\
\hline $\begin{array}{lll} & & \\
0 & 0 & 0\end{array}$ & $\%$ MnInHM & 61.1 & 58.89 \\
\hline 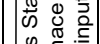 & $\%$ SilnHM & 21.35 & 22.6 \\
\hline 号 & $\% F e l n H M$ & 14.82 & 15.77 \\
\hline 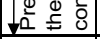 & SpPower & 4611.002 & 4419.999 \\
\hline ¿ & $\%$ MnInHM & 61.1 & 58.89 \\
\hline 营 & $\%$ SilnHM & 21.35 & 22.6 \\
\hline 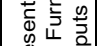 & $\%$ FelnHM & 14.82 & 15.77 \\
\hline$\stackrel{0}{5}$ & SpPower & 3739.002 & 3064.998 \\
\hline
\end{tabular}

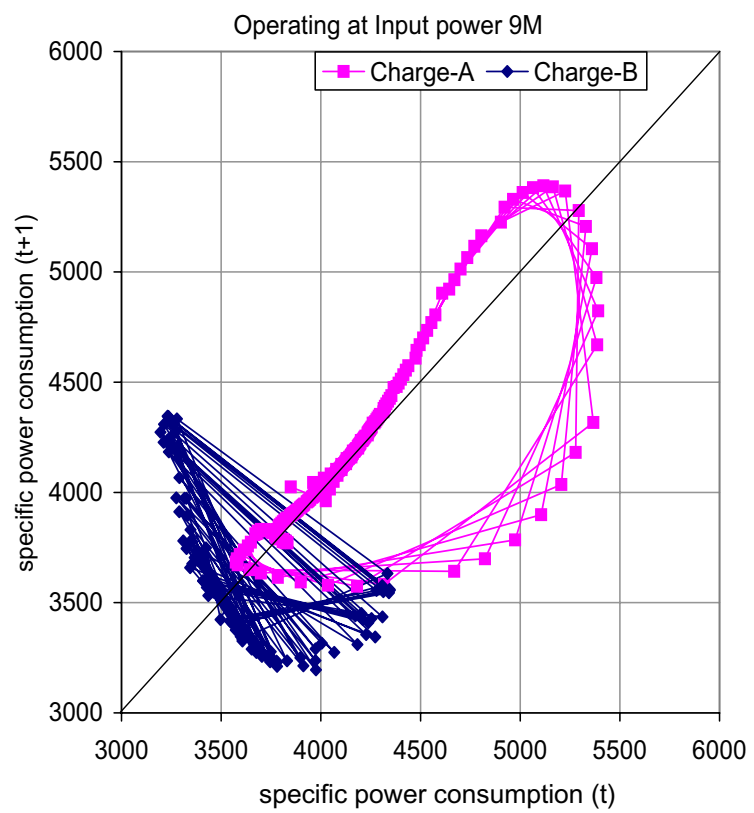

Fig. 16. Change in the SAF behaviour with changes in the input charge derived from the simulator developed. The charge-B shows lesser variation and lower specific power consumption when compared to charge-A in the plot.

done using only the parameters available for change and thus possible alternatives appear easily.

The inputs are in two parts, one is based on the charging to be done and the input power to be applied and the other is based on the output produced by furnace in the immediate past over which the new charging will be done as shown below.

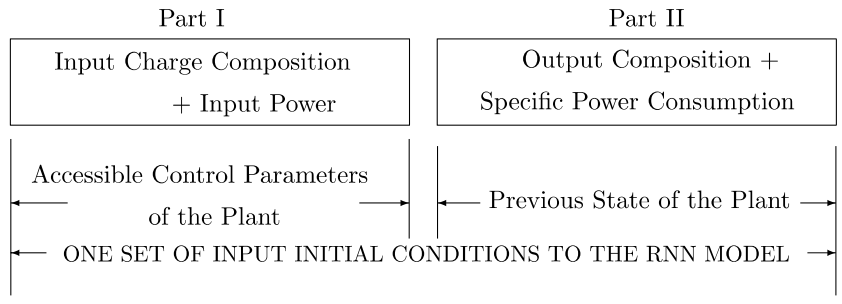

Thus, to reach the new input requirements, search has to be made taking the current outputs of the furnace into consideration. If the new input conditions selected are based on the immediate past output of the process then it can be implemented straightaway. In case it requires a different output conditions of the SAF over which it may be based upon, the intermediate conditions of the SAF operation can be searched using the Genetic Algorithms based procedure developed in the next section.
The SAF is a semi-batch process and only a part of the contents of the furnace is taken out at each tapping in the form of hot metal and thus if the initial charging conditions of raw material and power inputs are maintained long enough, the same outcome will be achieved at irrespective of the current outputs of the furnace over which the changes are being carried out. This suggests that the shape of final attractor of the SAF for any set of initial conditions as specified by raw materials and input power, is fixed but path to it will depend upon second portion of the initial conditions, specified by immediate past outputs of the SAF.

In the implementation example shown in Fig. 16 the current attractor (charge-A) of the process, obtained through calculations using the RNN model is plotted (for roughly one cycle) with respect to specific power consumption of the SAF. The range of variations can be seen from the figure, and also intercepts on a $45^{\circ}$ line on this figure shows the areas of maximum and minimum specific power consumptions.

The second attractor searched through visual approach through the furnace simulator is shown in Fig. 16 for charge-B. The input conditions for this have been arrived at through Genetic Algorithm based search (Section 5.3). This attractor as can be seen is more compact, and less variations in output parameters will take place if the pro- 
cess is switched onto this. Further, the region of operation of this attractor is in the zone where the specific power consumption is lower, which is a desirable condition.

While searching for the right inputs, care was taken in not selecting charges where values of any variable are changed by less than one percent, as these changes may be lost in the observation and control errors in the plant.

An important feature of this approach is that the desirable operating condition for the given attractor are searched off-line and only the final values have been implemented on the plant. Thus the objective can be achieved in a few steps and this makes it suitable for implementation on real-world systems.

However, a limitation of this approach may be the requirement that the initial condition may have to be varied significantly in search of a desirable attractor which may affect some other features of operations.

\subsection{Controlling a chaotic process with given inputs, with small changes during operation}

In real-world chaotic process plants often it is impractical to keep the input conditions constant during the operations and thus in building a chaotic process control model this should be taken into consideration. It follows from the fact that the condition of the plant and evolution gets redefined every time the changes are made in any of the inputs of the chaotic plant. This approach becomes relevant when the operating conditions of the plant are not far away from desirable ones generally needed to be maintained with the added objective of reducing the chaotic variations in the output.

The problem of reducing the variations in the output has been widely tackled in literature [16] by stabilising the chaotic orbits on to a fixed point of the systems and is relevant here. But the fact that inputs parameters continue to change does not allow standard efforts like those of Weeks and Burgess [17] and others to be implemented and hence a modified procedure outlined in [10] has been experimented with.

In this approach, using the given input values for the plant, the attractor of the process can be plotted using the RNN model and the subsequent values of the output parameter of the process can be seen. If the operating points are going away from the desirable zone, subsequent values of parameters can be altered by giving a series of suitably determined $( \pm)$ perturbations to accessible parameters of the plant without changing the basic input conditions and the attractor. The model permits small changes to be made in input parameters while the system is being driven towards the fixed point for eventual stabilisation.
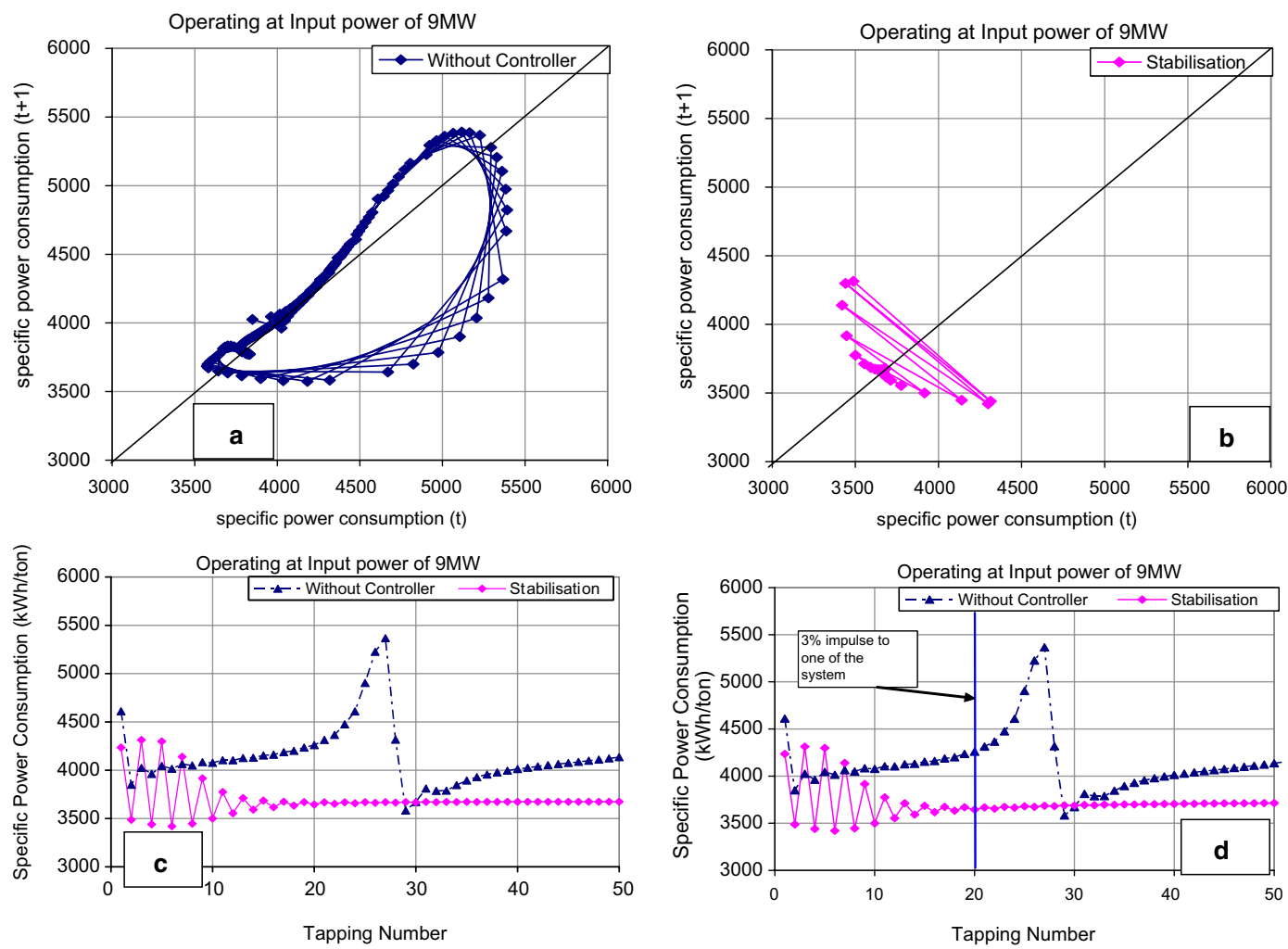

Fig. 17. Stabilisation simulation of the plant onto the unstable fixed point using RNN model of plant and GA: (a) Phase-space attractor of specific power consumption before stabilisation, (b) phase-space attractor of specific power consumption during stabilisation, (c) tapping by tapping plot during stabilisation and (d) tapping by tapping plot during stabilisation with sudden change in the initial conditions at tapping number 20 . 
Additional constraints while determining the suitable perturbations are the requirement that the process output values must not change into undesirable values during the implementation and the proposed input changes should be implementable (should not be lost in the noise of observation and implementation of the plant) on the process plant.

Experiments were performed on the MIMO-RNN based SAF simulator developed, (Appendix A) and tested for its capability to model chaos and predict variations due to small input changes. To test the plausibility of the proposal, a particular case of chaotic working of SAF shown in Fig. 17(a) through its attractor (computed using the RNN model) was selected for stabilisation on or near a fixed point on the attractor, using the procedure outlined above. This attractor is observed to have cycle of 29 steps. The simulation for the stabilisation was carried out with two different input cases. In the first case the inputs were kept constant throughout and in the second case they were altered once by about 3\% during the process. The impulses used for stabilisation were $2 \%$ of the parameter values so that they were not lost in the observation and implementation variations of the plant. Fig. 17(b) shows that a near stable working could be achieved in steps and the range of variation of specific power is reduced from $3700-5000 \mathrm{~kW} \mathrm{h/ton}$ to $3000-3200 \mathrm{~kW} \mathrm{h/ton.} \mathrm{Fig.} 17$ (c) shows same stabilisation in term of tapping number of the plant. Fig. 17(d) shows the stabilisation efforts on the attractor when the inputs are altered once. It can be seen that stabilisation is still achieved much in the same way.

The above experiments demonstrate the plausibility of using MIMO-RNN models for stabilising a realworld process plant in presence of plant noise and some changes in input parameters during the operation.

However, it can be observed that the procedure needs to be implemented on a real plant and if these are too many small steps by way of impulses it may become impractical and also undesirable. But it may also be noted that the number of steps needed may depend on the nature (e.g., presence of variety of attractors, etc.) of the chaotic system.

The above studies through the simulator of a realworld SAF show, the plausibility of stabilizing a real chaotic system through RNN-GA approach. The approach works even when some small changes occur in initial conditions during the stabilisation effort.

\section{Conclusions from the studies}

(1) It has been shown that low MSE RNN models for a chaotic system can be developed from short chaotic and noisy observed data of real-world processes such as a SAF. These models then can be used for predicting the future values of the process variables and constructing the bifurcation diagrams of the system, for control through input parameters.

(2) The MIMO features of the RNN models built provide easily accessible parameters for controlling the chaotic systems, through input controls and lead to alternatives in selecting the parameters, which can be changed through bifurcation diagram for any of the input parameters.

(3) It is observed that real-world chaotic systems like SAF have very rich sets of bifurcation diagrams and attractors and the process can be shifted to more described conditions of operation by changing from one attractor to another, through searches in input patterns using computational intelligence techniques with RNN models.

(4) Plausibility of stabilising a real-world chaotic system on an unstable fixed point in the chaotic regime, through small perturbations in accessible input parameters even in the presence of noise and small changes in input conditions during stabilisation, has been demonstrated using the computational intelligence techniques working on the MIMO-RNN model of the system.

\section{Acknowledgements}

The authors are thankful to the ferroalloy producing organisation which has provided data for this study, and also SERC-DST, Government of India which provided grants as per Sanction no. SR/FTP/ET-83/2001 under its scheme of Fast track proposal for young scientists.

\section{Appendix A. Submerged arc furnace (SAF)}

As the name implies in the submerged arc furnace the electrode is submerged inside the raw material (ores); and it employs electric arc within the furnace to generate large quantity of heat energy. The Fig. 18 shows a simple submerged arc furnace with three electrodes connected to a power source. The arc between the electrodes and raw materials induced once certain distance between them is maintained. The heat concentrates at the arc to develop high temperatures necessary for a high rate of heat transfer from the arc to the metal within the furnace. The main application of the submerged arc furnace is to produce the ferroalloys.

The ferroalloys are used as reducing agents in alloy steel production. There are wide varieties of ferroalloys namely ferromanganese, ferrochromium, ferrosilicon, silico-manganese, etc. The submerged arc furnace can be classified as complex system with its high temperature, huge electrical arcs with high currents, and multiple 


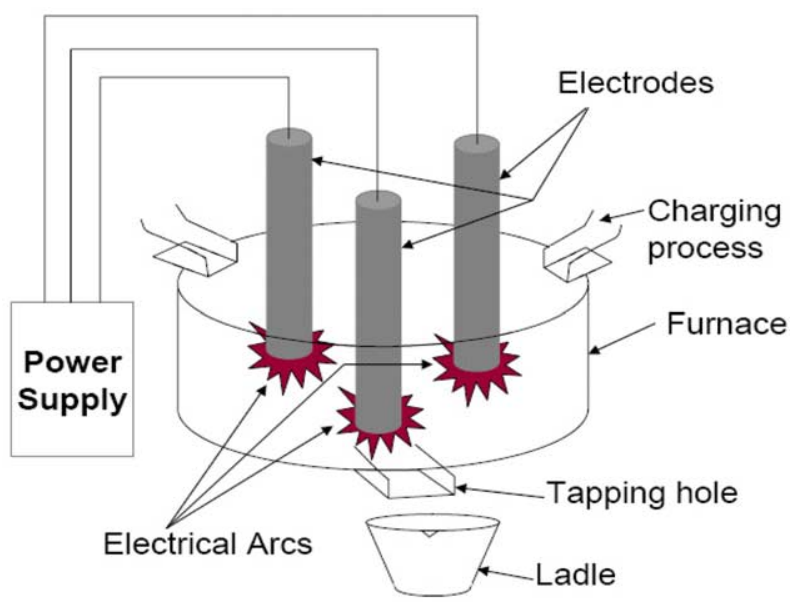

Fig. 18. A simple submerged arc furnace.

chemical reactions. A single chemical reaction can be understood individually, but the same dynamics is not true when reaction is performed with other chemical reactions i.e., a chemical reaction will behave differently when it is with other reaction rather alone. This is the reason why it is difficult to analyse or understand or model the total chemical reactions inside the furnace. In addition to the chemical reactions, a huge mass sits in side the furnace, which will be dictating the time varying reactions.

\section{References}

[1] C.B. SaiBabu, A hybrid intelligent system for modelling, control and optimization of large-scale industrial processes, Ph.D. thesis, Indian Institute of Technology, Kharagpur, India (December 1998).

[2] K.V. Rajaram, M.A. Faruqi, Development of intelligent system, for predicting sticker induced breakouts in continuous casting based on artificial neural networks, Final Report ME/1998/ TISCO/2, Indian Institute of Technology, Kharagpur, India (September 1998).
[3] S.K. Chand, Modeling of complex systems from time series data: a computational intelligent approach, Ph.D. thesis, Indian Institute of Technology, Kharagpur, India (January 2000).

[4] J.C. Sprott, Chaos and Time-series Analysis, Oxford University Press, NY, 2003.

[5] J. Kurths, B. Boccaletti, C. Grebogi, Y.-C. Lai, Introduction: control and synchronization in chaotic dynamical systems, Chaos 13 (1) (2003) 126-127.

[6] E. Ott, C. Grebogi, J.A. Yorke, Controlling of chaos, Physical Review Letters 64 (1990) 1192-1196.

[7] W.L. Ditto, S.N. Rauseo, M.L. Spano, Experimental control of chaos, Physical Review Letters 65 (26) (1990) 3211-3214.

[8] W.L. Ditto, L.M. Pecora, Mastering chaos: it is now possible to control some systems that behave chaotically engineers can use chaos to stabilize lasers, electronic circuits and even the hearts of animals, Scientific American 269 (1993) 78-84.

[9] A.G. de Oliveira, A.P. Tsui, A.J. Jones, Using a neural network to calculate the sensitivity vectors in synchronisation of chaotic maps, in: Proceedings 1997 International Symposium on Nonlinear Theory and its Applications (NOLTA'97), vol. 1, Research Society of Nonlinear Theory and its Applications, IEICE, Honolulu, USA, 1997, pp. 46-49.

[10] J. Krishnaiah, C. Kumar, M.A.Faruqi, Modelling and control of chaotic processes through their bifurcation diagrams generated with the help of recurrent neural networks models: Part 1simulation studies, Journal of Process Control, in press, doi:10.1016/j.jprocont.2005.04.002.

[11] J. Krishnaiah, C.S. Kumar, M.A. Faruqi, Constructing bifurcation diagram for a chaotic time-series data through a recurrent neural network model, in: ICONIP'02 Proceedings, 2002, pp. 2354-2358.

[12] F. Takens, Detecting strange attractors in turbulence: appeared in dynamical systems and turbulence, Lecture Notes in Maths, vol. 898, Springer-Verlag, Berlin, 1981, pp. 366-381.

[13] O. De Feo, Self-emergence of chaos in the identification of irregular periodic behaviour, Chaos 13 (4) (2003) 1205-1215.

[14] S. Gadaleta, G. Dangelmayr, Optimal chaos control through reinforcement learning, Chaos 9 (3) (1999) 775-788.

[15] S. Haykin, Neural Networks: A Comprehensive Foundation, second ed., Prentice-Hall, Englewood Cliffs, NJ, 1999.

[16] H. El-Faramawy, T. Mattar, A. Fathy, M. Eissa, A. Ahmed, Silicomanganese production from manganese rich slag, Ironmaking \& Steelmaking 31 (1) (2004) 31-36.

[17] E.R. Weeks, J.M. Burgess, Evolving artificial neural networks to control chaotic systems, Physical Review E 56 (2) (1997) 15311540 . 